\title{
Comment expliquer la forme non elliptique de l'amphithéâtre de Leptis Magna (Al Khums/Lybie) ?
}

Jean-Claude Golvin

\section{(2) OpenEdition \\ Journals}

Édition électronique

URL : http://journals.openedition.org/edl/123

DOI : $10.4000 /$ edl. 123

ISSN : 2296-5084

Éditeur

Université de Lausanne

\section{Édition imprimée}

Date de publication : 15 mai 2011

Pagination : $307-324$

ISBN : 978-2-940331-25-3

ISSN : 0014-2026

Référence électronique

Jean-Claude Golvin, "Comment expliquer la forme non elliptique de l'amphithéâtre de Leptis Magna (Al Khums/Lybie) ? », Études de lettres [En ligne], 1-2 | 2011, mis en ligne le 15 mai 2014, consulté le 19 décembre 2020. URL : http://journals.openedition.org/edl/123 ; DOI : https://doi.org/10.4000/edl.123 


\section{COMMENT EXPLIQUER LA FORME NON ELLIPTIQUE DE L'AMPHITHÉÂTRE DE LEPTIS MAGNA (AL KHUMS/LYBIE)?}

La forme générale de l'amphithéâtre de Leptis Magna, monument très bien conservé et daté avec précision, n'est pas elliptique et aucune explication n’a été donnée jusqu’à présent de cette situation particulière. Pourtant le monument, entièrement creusé dans le roc, aurait pu posséder la forme elliptique caractéristique de ce type d'édifice, comme c'est le cas pour les amphithéâtres de Cagliari ou de Syracuse. Nous avons émis l'hypothèse que la volonté manifeste de concevoir un monument dont le plan rappelle celui de deux théâtres accolés, plan qui ne se rencontre plus par la suite, devait être liée aux spectacles particuliers qui se déroulèrent sous le règne de Néron.

L’amphithéâtre de Leptis Magna est daté avec précision par son inscription dédicatoire qui permet de situer son inauguration dans le courant du premier semestre de l'année 56 apr. J.-C. sous le règne de Néron ${ }^{1}$. La caractéristique architecturale la plus surprenante de cet édifice très bien conservé est la forme peu courante de son plan qui, bien que constatée depuis longtemps, n'a jamais fait l'objet d'une tentative d'explication satisfaisante ${ }^{2}$ : tel est donc le but du présent article.

I. G. Di Vita-Evrard, «Les dédicaces de l'amphithéâtre et du cirque de Leptis Magna».

2. K. E. Welch, The Roman amphitheater. Dans cet ouvrage important relatif à l'évolution architecturale de l'amphithéâtre romain, l'auteur ne cite même pas le cas de Leptis Magna. Nous avons tenu à signaler cette omission car elle ne peut être fortuite de la part d'un auteur connaissant aussi bien ce type de monument: J.-C. Golvin, «K. Welch, The Roman amphitheater from its origins to the Colosseum». Il est en effet difficile de situer cet exemple dans l'évolution architecturale des amphithéâtres sans expliquer pour quelles raisons il présente une forme inhabituelle. 
Le monument comprend en effet deux extrémités semi-circulaires raccordées par de courtes parties rectilignes ${ }^{3}$ (fig. 1). Le plan ressemble ainsi à celui de deux théâtres que l'on aurait accolés l'un à l'autre par leurs bâtiments de scène (fig. 2). La forme générale n'est donc pas elliptique, contrairement à celle de presque tous les amphithéâtres connus à ce jour (plus de deux cents exemples) dont beaucoup existaient déjà avant l'année 56 de notre ère. Cette forme particulière est nettement perceptible sur les photographies du monument (fig. 3 à 5).

Ce n'est certainement pas le projet sans lendemain de Curion-le-Jeune, réalisé à Rome un siècle plus tôt, qui aurait pu inspirer directement l'architecte du monument de Tripolitaine ${ }^{4}$.

Par ailleurs, l'amphithéâtre de Leptis Magna ayant été entièrement creusé dans le roc, rien n'aurait empêché de lui donner une forme elliptique si on l'avait voulu et comme c'est effectivement le cas pour les autres monuments en grande partie excavés: Sutri, Syracuse, Cagliari ${ }^{5}$. Il faut donc que des raisons particulièrement importantes aient joué pour que ce monument n'ait pas adopté la forme courante de ce type d'édifice, qui était déjà celle de l'amphithéâtre de Pompéi dès 70 av. J.-C. ${ }^{6}$

Il est vain de chercher à expliquer l'adoption de la forme de l'amphithéâtre de Leptis Magna à l'appui d'exemples rares plus anciens ${ }^{7}$, mieux

3. Sur l'amphithéâtre de Leptis Magna: A. Di Vita, «Recenti scavi e scoperte in Tripolitania»; O. Mahjiub, A. Chigine, R. Madaro, "Nuove ricerche nell'anfiteatro di Leptis Magna".

4. Pline, Histoire Naturelle 36.24. Le mécanisme qui permettait aux deux théâtres de bois de se raccorder s'est rapidement détraqué. Cette solution spectaculaire, insolite et dangereuse n'a pas bien fonctionné et n’a jamais été reproduite.

5. Pour toutes les références à d'autres exemples et pour simplifier, nous renvoyons à l'ouvrage général: J.-C. Golvin, L'amphithéâtre romain. En ce qui concerne Sutri, p. 40 sq.; Syracuse, p. 115 sq. ; Cagliari, p. 208. Pour la description de l'amphithéâtre de Leptis Magna, p. 83 sq.

6. On peut penser que l'amphithéâtre de Pompéi est vraiment le premier monument en pierre doté d'une cavea et d'une arène elliptiques, sans doute à l'inspiration de la forme architecturale idéale que finit par adopter l'édifice provisoire en bois construit précédemment sur le Forum Romanum: J.-C. Golvin, "L'amphithéâtre de Pompéi, monument de transition?".

7. Le cas de l'amphithéâtre de Césarée de Maurétanie datant de l'époque de Juba II (début du I ${ }^{\text {er }}$ siècle apr. J.-C.) dont l'arène, très allongée, rappelle celle d'un petit stade est particulier: J.-C. Golvin, L'amphithéâtre romain, p. 112-114. Le seul autre exemple, antérieur à l'an 56 de notre ère et dont la forme s'éloigne quelque peu de l'ellipse, est l'amphithéâtre de Luceria: ibid., p. 76 sq. Ce monument étant daté de 2 av. J.-C., il ne 
Fig. 1 - Relevé des contours de l'amphithéâtre de Leptis Magna. 
Fig. 2 - Restitution d'ensemble du plan de l'amphithéâtre de Leptis Magna. 
Fig. 3 - Aspect de l'amphithéâtre de Leptis Magna montrant la forme non elliptique de la cavea, vue depuis le sud-ouest.

Fig. 4 - Aspect de la moitié nord de la cavea. 
Fig. 5 - Sur le mur de podium se distinguent les pierres creuses et régulièrement réparties servant à ancrer de biais les poteaux du filet qui était tendu en direction de l'arène.

vaut examiner les conditions particulières qui ont joué à l'époque même de sa création, celle de Néron.

Il est un fait que cet empereur voulut imposer, jusqu'à les rendre officiels en 60 apr. J.-C., des jeux particuliers appelés neronia ${ }^{8}$ qui seront supprimés aussitôt après sa mort. Ces jeux associaient aux munera et aux venationes romains, des compétitions athlétiques et hippiques, des danses et des concours musicaux de tradition grecque. On sait combien Néron était influencé par la culture grecque, mais aussi combien l'hellénisme était mal perçu à Rome. Un tel type de spectacle ne pouvait exister que par la volonté de cet empereur et ne lui a pas survécu. Il est évident que

peut pas avoir influencé directement celui de Leptis Magna; on ne verrait d'ailleurs pas pourquoi.

8. A ce sujet: J.-P. Thuillier, Le sport dans la Rome antique, p. 167 sq. Suétone, Nero 12, mentionne les munera, les venationes, la naumachie ainsi que les pyrrhiques dansées par des éphèbes, les représentations de scènes de la mythologie qui étaient en réalité des exécutions mises en scène (mythes de Pasiphae et d'Icare) et les concours quinquennaux suivant l'usage grec: musicaux, gymniques, hippiques. Dans son texte, ces joutes néroniennes sont appelées neronia. 
Fig. 6 - Plan schématique d'ensemble montrant l'amphithéâtre (A), le premier état supposé du cirque qui pourrait remonter à l'époque de Néron, indiqué en pointillé (C1) et le second état du cirque daté du II e siècle apr. J.-C. (C2). L'amphithéâtre et le cirque sont reliés par deux corridors souterrains et par deux espaces à ciel ouvert qui aboutissent aux grandes entrées donnant sur l'arène.

si les neronia finirent par être officialisés en 60, l'idée de les donner était née quelques années plus tôt et tout porte à croire qu'elle avait été déjà mise en pratique dès les années 56-57 de notre ère.

La forme particulière de l'amphithéâtre de Leptis Magna s'expliquerait, à notre sens, par la vocation nouvelle de l'édifice: celle d'un monument polyvalent propre au déroulement de spectacles qui rappelaient pour partie ceux de l'amphithéâtre, du stade, du théâtre et du cirque. Il faut noter aussi que l'amphithéâtre de Leptis Magna fut sans doute, dès l'origine, relié au cirque voisin dans son premier état (fig. 6) et que l'on disposait ainsi d'un complexe monumental particulièrement complet ${ }^{9}$. Si les neronia ont bien eu un rapport direct avec la création de ce type d'édifice, le cas de Leptis Magna mérite d'être rapproché de Rome où Néron fit construire sur le Champ de Mars, en moins d'une année, un amphithéâtre en bois à une date très proche puisque l'on peut dater ce

9. Cette hypothèse, qui permet de bien expliquer certaines caractéristiques du cirque de Leptis Magna, a été émise par F. Fauquet, Le cirque romain, essai de théorisation de sa forme et de ses fonctions et L. Maurin, "Les édifices de cirque en Afrique». 
projet de 57 apr. J.-C. ${ }^{10}$ Moins d'un an sépare les deux réalisations et, si le monument de Rome (probablement victime de l'incendie de 64) n'a pas laissé de trace, les textes anciens en illustrent bien les caractéristiques architecturales et le fonctionnement. Ainsi, un des exemples semble éclairer l'autre et seuls des impératifs fonctionnels majeurs peuvent expliquer la ressemblance des deux édifices.

Dion Cassius révèle que l'amphithéâtre de Rome avait un sous-sol perfectionné et l'on peut se demander si celui de Leptis Magna n'en rappellerait pas la disposition ${ }^{11}$. Malheureusement ce dernier n'a pas encore été entièrement dégagé et des détails qu'il serait intéressant de commenter restent à découvrir. Cependant, sa disposition générale se devine à l'observation des vestiges visibles depuis l'arène (fig. 3 et 4). Il comprenait deux galeries de distribution, l'une située sur le grand axe, qui devait être en relation avec l'extérieur et une autre sur le petit axe (fig. 2). A leur intersection se trouvait une grande pièce centrale de plan presque carré, couverte par un plancher. Sur les galeries s'observent aussi les trappes de sortie des monte-charges. Ces dispositions montrent que ce monument permettait de réaliser des changements de décor à vue et des effets scéniques dont parlent les textes anciens. Suétone et Dion Cassius mentionnent que, lors de l'inauguration de l'amphithéâtre de Rome, une naumachie fut donnée dans un décor approprié ${ }^{12}$, mais rien ne prouve aujourd'hui que l'amphithéâtre de Leptis Magna ait disposé d'installations hydrauliques. La chose reste cependant possible et des fouilles permettront un jour de le vérifier. Rien ne dit que les naumachies aient été de grandes dimensions. On pouvait faire sortir par les monte-charges et les planchers mobiles un décor adéquat (paysage nilotique ou maritime) avec une maigre quantité d'eau dans des bassins peu profonds où pataugeaient des animaux aquatiques (crocodiles, phoques, hippopotames...).

Io. L'amphithéâtre de Néron est évoqué par: Tacite, Annales 13.31, qui précise qu’il fut construit quand Néron était consul pour la seconde fois avec Pison pour collègue; Pline, Histoire Naturelle 16.200, évoque le velum azur parsemé d'étoiles des amphithéâtres de l'empereur Néron et donc celui des édifices de spectacles de ce règne; pour Suétone, Nero 12, l'amphithéâtre de bois a été construit en moins d'une année dans la région du Champ de Mars mais son emplacement exact, peut-être proche des Saepta Iulia, ne peut être prouvé; le monument est enfin évoqué par Aurelius Victor, Liber de Caesaribus 5.3.

II. Dion Cassius 61.9.5 et 62.15.1.

I2. Suétone, Nero 12; Dion Cassius 61.9.5 et 62.15.1. 
Enfin et bien que la chose soit possible, rien ne prouve que le sous-sol de Leptis Magna ait existé dès l'origine. Il existe en effet des cas où le soussol n’a été réalisé qu'après coup, comme à Mérida ${ }^{13}$.

La forme générale du monument vu par le berger Corydon qu'évoque Calpurnius Siculus mérite d'être analysée attentivement. En effet, si on suit le texte à la lettre, on apprend que les courbes de l'arène incurvée entourent une surface plane, dont le milieu, relié «à une double masse", a la forme d'un œuf ${ }^{14}$. L'évocation d'une "double masse» n'aurait pas lieu d'être si le monument avait été elliptique, car il aurait été perçu d'un seul tenant alors qu'elle correspondrait effectivement à celle d'un monument ressemblant à deux théâtres dont les «masses» seraient accolées. La forme de l'arène bien que non elliptique aurait néanmoins grossièrement un aspect ovoïde et correspondrait bien au tracé du plan de Leptis Magna. Ce détail indique, à notre sens, que l'auteur ne décrit pas un amphithéâtre de forme elliptique, mais qu'il essaie de nous évoquer l'effet que produisait la forme particulière du monument décrit. Cet édifice ne peut donc pas être le Colisée de Rome, le plus vaste des amphithéâtres elliptiques qui apparaîtra à l'époque flavienne. De quel amphithéâtre s'agit-il donc? Nous ne connaissons pas la forme exacte du seul autre amphithéâtre qui existait à Rome à l'époque de Néron, celui de Statilius Taurus ${ }^{15}$, mais il est peu vraisemblable que le texte évoque ce monument, daté de l'époque augustéenne, qui a été dédaigné par les empereurs suivants ${ }^{16}$. En revanche, l'amphithéâtre de Néron a toujours été vanté comme une œuvre exceptionnelle. Enfin, la richesse de la décoration et les perfectionnements de l'amphithéâtre évoqués par Calpurnius Siculus correspondent bien aux caractéristiques que les autres auteurs anciens attribuent expressément à l'amphithéâtre de Néron. En fin de compte, tout porte à croire que le monument évoqué par Calpurnius

13. A propos de Mérida: J.-C. Golvin, L’amphithéâtre romain, p. 109 sq.

I4. Calpurnius Siculus, Bucoliques 7.30.

15. Sur l'amphithéâtre de Statilius Taurus, on se reportera en dernier lieu à K. E. Welch, The Roman amphitheater, p. 108-127. Tout porte à croire que la conception de cet édifice a suivi l'évolution architecturale d'ensemble des amphithéâtres depuis la construction de celui de Pompéi. Le monument était donc certainement elliptique.

I6. Suétone, Caligula 21. Cet empereur ne voulut pas utiliser l'amphithéâtre de Taurus et il commença la construction d'un amphithéâtre près des Saepta Iulia dont la réalisation fut abandonnée par son successeur Claude. 
Siculus est bien celui de Néron puisqu'aucune autre hypothèse n’est satisfaisante.

La hauteur du mur de podium est de 2,85 m à Leptis Magna. Elle est donc assez faible pour qu'il ait été utile de la surmonter, dès l'origine, d'un filet de protection supplémentaire qui aurait pu être restauré par la suite. Calpurnius Siculus évoque à propos du monument de Rome un filet tissé d'or tendu vers l'arène sur des défenses d'éléphant entières, toutes égales ${ }^{17}$. L'expression "tendu vers l'arène» conviendrait à décrire le filet de Leptis Magna puisque celui-ci était, comme on peut le constater sur le site, porté par des supports obliques plantés dans des trous au sommet du mur de podium tous les 2,40 m (fig. 5). Le filet s'avançait donc bien vers l'arène et au-dessus d'elle (fig. 2) et il convient de dire que cette disposition n'existe dans aucun des amphithéâtres elliptiques connus à ce jour.

On peut donc se risquer à restituer par hypothèse l'amphithéâtre de bois de Néron en s'inspirant des caractéristiques de celui de Leptis Magna. Il est certain que le monument de Rome était de grandes dimensions si l'on en croit Calpurnius Siculus ${ }^{18}$. Il ne devait certainement pas être plus petit que le monument de Tripolitaine dont l'arène mesure $57,20 \mathrm{~m} \times 47,30 \mathrm{~m}$ et la cavea $121 \mathrm{~m} \times 111 \mathrm{~m}$ et il ne devait pas comporter moins de gradins (fig. 2). Il possédait une galerie supérieure à portique, mentionnée par Calpurnius Siculus. Notons qu'une galerie à demi-colonnes engagées, dont de nombreux éléments ont été retrouvés épars, couronnait la cavea à Leptis Magna. On ne sait pas si un temple existait à Rome en haut de la cavea comme c'est le cas dans la grande cité de Tripolitaine (fig. 2). Nous en avons figuré un qui pourrait s'insérer dans la partie rectiligne du plan et contribuer par sa présence à créer une coupure visuelle. Celle-ci contribuerait fortement à donner à un observateur l'impression de voir deux masses situées de part et d'autre comme en témoigne le texte de Calpurnius Siculus (fig. 7). On ne voit pas pourquoi ni comment cet auteur aurait inventé cette forme insolite s'il ne l'avait pas vue. Le monument qu'il fait décrire à Corydon ne pouvait être qu'un édifice qu'il avait observé lui-même et dont le caractère

17. Calpurnius Siculus, Bucoliques 7.55.

18. Le berger Corydon voit se dresser jusqu'au ciel un amphithéâtre aux poutres enchevêtrées qui dominait presque la roche Tarpéienne avec des gradins immenses aux pentes légèrement inclinées: Calpurnius Siculus, Bucoliques 7.25. 
Fig. 7 - Restitution hypothétique de l'aspect général de l'amphithéâtre de bois construit pas Néron en 57 apr. J.-C. sur le Champ de Mars.

extraordinaire l'avait frappé. Evidemment, en vantant les mérites de cet édifice, il ne pouvait que satisfaire l'empereur qui l'avait créé et ce dernier ne peut être que Néron.

Lauteur souligne aussi la splendeur de la décoration, les pierres précieuses et l'or décorant galerie et portique. La magnificence de cette décoration compensait la modicité du matériau de base employé (le bois) qui fut choisi sans doute pour permettre une réalisation très rapide. Le monument put ainsi être achevé "en moins d'une année» comme le précise Suétone. La construction de grands édifices de spectacle en bois ne posait aucun problème à Rome. C'était au contraire une longue tradition illustrée par les édifices amovibles montés sur le Forum Romanum ${ }^{19}$ ou des réalisations particulières et mémorables comme le théâtre de Curion ou le theatrum et proscaenium ad Apollinis ${ }^{20}$ sur le Champ de Mars qui

19. Au sujet de ces édifices et de leur restitution possible: K. E. Welch, The Roman amphitheater, p. 30-71.

20. Tite-Live, Histoire romaine 40.51.3. 
précéda le théâtre de Marcellus. Mais c'est surtout le souvenir du luxe extraordinaire du théâtre de Marcus Scaurus qui nous vient à l'esprit et nous semble digne d'être comparé à l'amphithéâtre de Néron ${ }^{21}$. L'aspect extérieur des amphithéâtres de bois nous est aussi donné par certains reliefs de la colonne Trajane.

Il faut imaginer une grande structure faite de poutres entrecroisées pour assurer le contreventement de l'édifice. Elles devaient comprendre des attaches métalliques très solides pour éviter la catastrophe survenue à Fidènes sous Tibère où un amphithéâtre hâtivement conçu s'était effondré en faisant des milliers de victimes ${ }^{22}$.

Le mur de podium de l'amphithéâtre de Rome était en marbre (ou du moins revêtu de ce matériau) selon Calpurnius Siculus ${ }^{23}$ et, bien entendu, il nous faut restituer les cordages du grand velum dont l'existence est attestée par Pline, à défaut de tendre les voiles de lin azuré parsemées d'étoiles, pour ne pas cacher sur notre image l'intérieur du monument (fig. 7).

En fin de compte, les caractéristiques architecturales de l'amphithéâtre de Leptis Magna nous aident à restituer une image crédible de celui de Néron et les textes anciens relatifs à ce dernier offrent un moyen d'expliquer la forme du grand monument tripolitain.

A défaut de l'interprétation que nous venons de donner, aucune hypothèse ne nous semble pouvoir être avancée. Il n'y avait donc pas de raison de laisser plus longtemps inexpliquée la forme d'un des amphithéâtres les mieux conservés du monde ou d'omettre de le mentionner ni de tenter de nous donner une image crédible de l'étonnant édifice de Rome.

Ceci est bel et bien, mais une question fondamentale reste posée. Si ces monuments ont adopté cette forme pour des raisons fonctionnelles, quels en étaient les avantages réels?

Il faut en effet dire en quoi cette forme particulière répondait mieux aux exigences des neronia que la forme elliptique: tel est bien le seul moyen de la comprendre et de l'expliquer.

2I. Le théâtre de Marcus Scaurus daté de 58 av. J.-C. est évoqué par Pline, Histoire Naturelle 36.114.

22. L'amphithéâtre de Fidènes est évoqué par Tacite, Annales 4.62-63, et Suétone, Tiberius 40 et Caligula 31.

23. Calpurnius Siculus, Bucoliques 7.45. 
Le premier avantage qui nous paraît être évident ressort de l'observation du plan (fig. 2). Il est déterminant car il concerne les qualités acoustiques de la cavea. En effet, si le problème de l'acoustique était peu important dans les amphithéâtres elliptiques et les cirques ${ }^{24}$, il était fondamental dans le cas d'un monument destiné à des concours musicaux. Il est certain que dans les neronia, cette fonction n'était pas négligeable. On sait combien Néron accordait d'intérêt à ce type de spectacle puisqu'il allait jusqu'à se produire lui-même sur scène ${ }^{25}$. Pour que l'édifice ait les meilleures qualités acoustiques possible, il fallait que sa forme respecte celle de l'édifice idéalement conçu en fonction de cette exigence, le théâtre. C'est dire que sa cavea devait être de forme semi-circulaire (et non elliptique). Vitruve a beaucoup insisté sur le fait que la forme générale du théâtre devait se fonder sur les exigences de l'acoustique et que, pour cette raison, elle devait être semi-circulaire ${ }^{26}$. Les études scientifiques contemporaines menées sur le sujet l'ont prouvé ${ }^{27}$. La diffusion du son était idéale pour les deux parties semi-circulaires de l'amphithéâtre de Leptis Magna (ou de Rome) et elle n'était pas mauvaise dans les parties rectilignes car elles étaient très proches du lieu d'émission. Un spectacle à caractère musical donné au centre de l'arène était par conséquent très bien perçu. Bien entendu, on pouvait concevoir la disposition des musiciens et l'organisation de ce spectacle en fonction du plan particulier de l'édifice pour lequel la partie centrale de l'arène correspondait en quelque sorte à celle des scènes de deux théâtres accolés.

Le deuxième avantage était que la forme du plan ressemblait aussi un peu à celle d'un petit stade. Elle était ainsi mieux adaptée au déroulement de compétitions athlétiques qu'une arène elliptique. L'espace était suffisant pour la plupart des épreuves athlétiques (saut en longueur, lancé du disque et du javelot) et des sports violents (boxe, lutte, pancrace) qui étaient fort appréciés des Romains. La longueur de l'arène était courte pour la course de vitesse à pied, mais envisageable malgré tout. Une

24. On sait que l'acoustique était relativement mauvaise dans l'amphithéâtre elliptique: J.-C. Golvin, L'amphithéâtre romain, p. 341-344. En ce qui concerne le cirque: J. Nelis-Clément, "Le cirque romain et son paysage sonore».

25. Sur le goût immodéré de Néron pour la musique et le fait de se donner en spectacle: Suétone, Nero 20, 21 et 23.

26. P. Gros, "Le schéma vitruvien du théâtre et sa signification dans le système normatif du De Architectura" et L'architecture romaine, p. 278-280.

27. F. Canac, L'acoustique des théatres antiques. 
course plus longue imposait de tourner en avant du mur de podium, chose qui nous parait être possible puisqu'il en est de même aujourd'hui dans les stades. Les dimensions relativement réduites de l'arène par rapport à celles de la piste d'un véritable stade avaient pour avantage de permettre au public de voir les athlètes de près.

Un troisième avantage était de permettre de donner un spectacle extrêmement varié dans un cadre approprié car ce dernier convenait également bien aux munera et aux venationes. En effet, cette arène n'était pas mauvaise. Elle rappelait par sa forme celle de l'édifice d'origine en bois appelé spectacula que l'on avait si souvent monté occasionnellement sur le Forum Romanum avant l'invention de l'arène elliptique.

Le monument servait enfin à des spectacles hippiques. Il pouvait s'agir de courses de cavaliers, mais aussi de courses acrobatiques de desultores. On pouvait aussi faire évoluer les chevaux de façon savante comme dans les carrousels et des pyrrhiques étaient données par des jeunes gens. Aux intermèdes du spectacle pouvaient se produire bouffons, dresseurs et jongleurs.

La polyvalence de cette arène était excellente et l'existence du sous-sol lui permettait de fonctionner en continu en offrant les spectacles les plus variés avec des changements de décor à vue, évoqués notamment par Calpurnius Siculus.

Enfin, dans le cas de Leptis Magna, le lien étroit de l'amphithéâtre et du cirque permettait encore certainement d'autres combinaisons (fig. 6). Une pompa extraordinaire pouvait se préparer dans le cirque puis défiler dans l'arène et rien n'aurait empêché de faire passer des chars et de voir les cochers des factions annonçant les courses qui seraient données ensuite dans le cirque voisin.

Un autre point important est à considérer. Néron voulait imposer un nouveau type de spectacle et rien ne permettait de le faire plus efficacement que la conception d'un type de monument nouveau spécialement étudié à cette fin. Non seulement l'édifice était plus performant sur le plan fonctionnel, mais sa signification était renforcée. Il devenait le symbole de la nouvelle formule de spectacle et l'œuvre originale de l'empereur. Le fait que Néron ait voulu créer un édifice d'un type nouveau n'a rien d'étonnant puisque cet empereur s'est particulièrement distingué par ses innovations architecturales et ses projets inédits. Cette formule architecturale était efficace et, si elle n'a pas subsisté, sa fin s'explique par la suppression brutale des neronia. Leur composante hellénique, mais 
aussi leur lien avec le nom d'un empereur frappé d'infamie ont nui à leur maintien. Cette création architecturale ne pouvait que subir le sort des autres réalisations emblématiques du règne de Néron, telles que la Maison Dorée, à savoir l'effacement. Le bel amphithéâtre de bois disparut dès 64 apr. J.-C. dans le grand incendie de Rome et, bien entendu, aucun édifice de ce type ne fut construit après ce règne. En revanche, l'amphithéâtre de Leptis Magna, qui était creusé en grande partie dans le roc, a subsisté sans que son architecture ne puisse être modifiée. Même s'il ne servait plus de cadre à un spectacle tel que les neronia, il restait tout à fait efficace pour le déroulement des munera et des venationes. Si notre hypothèse est la bonne, ce monument offrirait pour nous l'intérêt d'avoir pérennisé l'aspect d'un type d'édifice particulier en figeant dans la pierre une formule architecturale qui, à notre sens, fut aussi celle du grand édifice de bois de Rome. Elle fut peut-être aussi celle d'autres monuments construits sous ce règne qui restent à découvrir. Il serait en effet particulièrement intéressant de savoir si d'autres amphithéâtres réalisés sous le règne de Néron auraient eux aussi cette forme particulière; on ne peut l'affirmer à ce jour. Un très grand nombre d'amphithéâtres restant à découvrir, on peut espérer le prouver.

Le seul but du présent article était de tenter d'expliquer l'origine de la forme insolite de l'amphithéâtre de Leptis Magna et sa très probable ressemblance avec celui de Néron à Rome. Il n'était pas d'étudier plus en détail la restitution architecturale de l'un et l'autre de ces édifices. Ce vaste et riche sujet qui est en cours d'étude fera l'objet de prochains articles.

Jean-Claude Golvin Institut Ausonius, Université de Bordeaux III 


\section{BIBLIOGRAPHIE}

\section{Sources}

Aurelius Victor, Liber de Caesaribus, Paris, Les Belles Lettres, 2003 (1975).

Calpurnius Siculus, Bucoliques, Paris, Les Belles Lettres, 2003 (1991).

Dion Cassius, Roman history, Books 61-70 (Loeb Classical Library 176), London, Loeb Classical Library, 1925.

Pline l'Ancien, Histoire naturelle, Livre XVI, Paris, Les Belles Lettres, 1962.

—, Histoire naturelle, Livre XXXVI, Paris, Les Belles Lettres, 1981.

Suétone, Vie des douze Césars, T. II: Tibère, Caligula, Claude, Néron, Paris, Les Belles Lettres, 2010.

Tacite, Annales, Livres XIII-XVI, Paris, Les Belles Lettres, 2010.

Tite-Live, Histoire romaine, Livre XL, Paris, Les Belles Lettres, 1986.

\section{Etudes}

Canac, François, L'acoustique des théâtres antiques, Paris, CNRS, 1967.

Di VITA, Antonino, "Recenti scavi e scoperte in Tripolitania», suppléments de Libya Antiqua, 2 (1966), p. 85-91.

Di Vita-Evrard, Ginette, «Les dédicaces de l'amphithéâtre et du cirque de Leptis Magna", Libya Antiqua, 2 (1965), p. 29-37.

FAUQuet, Fabricia, Le cirque romain, essai de théorisation de sa forme et de ses fonctions, thèse de doctorat soutenue à l'Université de Bordeaux III, 2002 (en cours de publication).

Golvin, Jean-Claude, L'amphithéâtre romain. Essai sur la théorisation de sa forme et de ses fonctions, Paris, De Boccard, 1988.

—, "L'amphithéâtre de Pompéi, monument de transition?», Nikephoros, 20 (2007), p. 199-207. 
-, "K. Welch, The Roman amphitheater from its origins to the Colosseum", Nikephoros, 20 (2007), p. 295-300.

Gros, Pierre, "Le schéma vitruvien du théâtre et sa signification dans le système normatif du De Architectura", Revue Archéologique, 1 (1994), p. 57-80.

—, L'architecture romaine, vol. 1, Paris, Picard, 1996.

Mahjiub, Omar, Chigine, Antonio, Madaro, Raffaele, "Nuove ricerche nell'anfiteatro di Leptis Magna", Libya Antiqua, 13 (1976-1977), p. 21-36.

Maurin, Louis, "Les édifices de cirque en Afrique: bilan archéologique", in Le cirque romain et son image, éds Jocelyne NelisClément, Jean-Michel Roddaz, Bordeaux, Ausonius, 2008, p. 91-108.

Nelis-Clément, Jocelyne, «Le cirque romain et son paysage sonore», in Le cirque romain et son image, éds Jocelyne Nelis-Clément, JeanMichel Roddaz, Bordeaux, Ausonius, 2008, p. 431-458.

Thuillier, Jean-Paul, Le sport dans la Rome antique, Paris, Errance, 1996.

WeLCH, Katherine E., The Roman amphitheater: from its origins to the Colosseum, New York, Cambridge University Press, 2007.

\section{Crédits iconographiques}

Fig. 1:

Mahjiub, Omar, Chigine, Antonio, Madaro, Raffaele, "Nuove ricerche nell'anfiteatro di Leptis Magna", Libya Antiqua, 13 (19761977), p. 24, fig. 1.

Fig. 2-7:

J.-C. Golvin. 
